\title{
Effects of 12-week combined exercise program on self-efficacy, physical activity level, and health related physical fitness of adults with intellectual disability
}

\author{
Garam Jo1 , Brenda Rossow-Kimball², Yongho Lee ${ }^{1, *}$ \\ 'Department of Physical Education, Seoul National University, Seoul, Korea \\ ${ }^{2}$ Faculty of Kinesiology and Heath Studies, University of Regina, Regina, SK, Canada
}

The current study examined the effects of an exercise program on health related physical fitness, self-efficacy, and physical activity levels in adults with intellectual disability. The study used pre- and posttest experimental research design with a control group. Total of 23 adults with intellectual disability were recruited with 12 assigned for the exercise group and 11 for the control group, separately. The measures of health related physical fitness included cardio pulmonary endurance (step-test), body composition (bioelectrical impedance analysis), flexibility (sit and reach), muscle endurance (sit-up), and strength (hand grip strength). Self-efficacy was measured using the physical self-efficacy scale. Accelerometers were used to measure physical activity levels.
All variables were measured and evaluated twice at baseline and at the end of the program. The exercise program consisted of band exercises and rhythmic activity for 90 min, twice per week for 12 weeks. After the intervention, significant improvements were found in the experimental group in muscle endurance, self-efficacy, and physical activity levels. An exercise program may be recommended as a nonpharmaceutical method to improve the health of adults with intellectual disabilities.

Keywords: Adapted physical activity, Self-efficacy, Physical fitness, Adults with intellectual disability, Physical activity level

\section{INTRODUCTION}

Physical activity (PA) is not only effective in the reduction of coronary artery disease risk factors and improvement of cardiovascular and respiratory muscle function, but also in the lowering of anxiety and depression, and the enhancement of psycho-emotional well-being and quality of life (Franklin et al., 2000). Unfortunately, the PA and physical fitness conditions of those with intellectual disability (ID) is very low compared with the general population (King and Mace, 1990; Lotan et al., 2004) for various reasons, including psychological and physiological factors (Fernhall and Tymeson, 1988) such as a passive lifestyle and low motivation.

Because school-aged children or youth with ID have been the main targets of school- or community-based exercise programs, adults with ID have naturally been marginalized from PA pro- grams in the Korean setting (Kang and Kim, 2010). The PA of adults with ID is especially serious, with low activity levels reported to be similar to those in the elderly population (Dixon-Ibarra et al., 2013). Due to their physical inactivity, adults with ID suffer from a variety of pathological and physiological problems such as heart disease, low fitness levels and muscle tension (Calders et al., 2011), which can lead to an increased level of overall mortality and morbidity.

Social cognitive theory (SCT) provides a framework for comprehending why people choose to engage in and sustain healthy behavior such as PA (Stacey et al., 2015). SCT considers human behavior to be a result of a dynamic and reciprocal interaction between personal, behavioral, and environmental influences (Bandura, 1986). Individuals believe that they can influence their own behavior and surrounding environment with purposeful and goal
${ }^{*}$ Corresponding author: Yongho Lee (10 https://orcid.org/0000-0002-6430-5327 Department of Physical Education, Seoul National University, 1 Gwanak-ro, Gwanak-gu, Seoul 08826, Korea

Tel: +82-2-880-7762, Fax: +82-2-872-2867, E-mail: gophers@snu.ac.kr Received: December 12, 2017 / Accepted: February 4, 2018
This is an Open Access article distributed under the terms of the Creative Commons Attribution Non-Commercial License (http://creativecommons.org/licenses/by-nc/4.0/) which permits unrestricted non-commercial use, distribution, and reproduction in any medium, provided the original work is properly cited. 
directed actions which, ultimately, influences future behaviors (Allen, 2004).

The detailed primary components of SCT are as follows: (a) understanding of health risk and benefits; (b) self-efficacy belief that a person can control their own health habits; (c) the expected costs and benefits or outcome expectations; (d) health goals, both proximal and distal intentions to engage in the behavior; (e) perceived facilitators and social support; and (f) barriers to making changes (Bandura, 2004). Self-efficacy, which is a person's belief in his or her ability to carry out a certain behavior successfully, is a key construct of SCT (Allen, 2004). Self-efficacy is theorized to have a direct effect on behavior initiation and cessation, effort and persistence, motivation, thought patterns, and emotional reactions (Bandura, 1997). Self-efficacy affects behavior directly, through belief in one's ability to apply skills effectively in difficult situations, as well as indirectly through effects on goals, outcome expectations, barriers and facilitators (Bandura, 1998; Bandura, 2004). Self-efficacy enables people to acquire knowledge and develop skills; as self-efficacy improves, individuals expect positive consequences, overcome barriers, and show motivation and commitment to goals (Bandura, 1998). In relation to people with ID, self-efficacy is the most important intrinsic motivation for overcoming various internal or external environmental limitations (Glanz et al., 2008) thus allowing them to participate actively in PA.

According to previous studies, the SCT framework explains $40 \%-71 \%$ of the variance in the PA behavior of adults (Anderson-Bill et al., 2011; Phillips and McAuley, 2013; Rovniak et al., 2002; White et al., 2012). Peterson et al. (2008), who confirmed a significant correlation between self-efficacy and PA, also emphasized the importance of self-efficacy for maintaining healthy habits. In line with these studies, Song and Jeong (2011) reported a significant increase of self-efficacy for school-aged children with ID through regular participation in PA programs. Because physical self-efficacy increases through improved physical function and subjective cognitive processes for learning exercise skills and mastery experience, providing regular support for adults with ID through a well-planned exercise training program could promote daily PA behavior in adults with ID.

Unfortunately, due to the limitations in the intellectual ability of adults with ID and within the research environment, the research carried out to date is limited. Specifically, most of the related studies employed cross-sectional survey designs using questionnaires to assess PA behavior. This has limitations in that changes in physical self-efficacy according to exercise participation cannot be observed and only a simplistic relationship can be inferred. Additionally, the reliability of the measured PA level is poor. Another limitation of previous studies is that participants were mainly people without disabilities or school-aged children or adolescents with ID, and the exercise intervention tools employed were expensive, heavy and difficult to apply easily to real environments.

The purpose of the present experimental exercise intervention study was to examine improvements in self-efficacy, PA levels, and physical fitness of adults with ID following an exercise intervention program. The program, designed in consideration of the subjects' traits, consisted of band strength training and music-based aerobic rhythmic activities.

\section{MATERIALS AND METHODS}

\section{Participants}

Participants for the current study were recruited from A Community Welfare Center, located in South Korea. Participants were required to satisfy the following inclusion criteria for the participants were as follows: (a) 18 years of age or older, (b) IQ (intelligence quotient) score between $45-70$, (c) able to engage in reciprocal communication with the researchers, and (d) no difficulty with PA requiring musculoskeletal movement. A total of 12 adults with ID were recruited and assigned for the exercise group; 11 adults were recruited for the control group (CG) separately due to limitations of the research environment. The study was ap-

Table 1. Participant characteristics

\begin{tabular}{lccrc}
\hline Variable & EG $(\mathrm{n}=10)$ & $\mathrm{CG}(\mathrm{n}=10)$ & $t$ & $P$-value \\
\hline Sex & & & & \\
$\quad$ Men & 7 & 3 & & \\
$\quad$ Women & 3 & 7 & & \\
Age $(\mathrm{yr})$ & $30.60 \pm 11.00$ & $31.80 \pm 11.32$ & -0.240 & 0.813 \\
Height $(\mathrm{cm})$ & $160.88 \pm 9.25$ & $161.34 \pm 10.93$ & -0.102 & 0.920 \\
Weight $(\mathrm{kg})$ & $59.79 \pm 14.46$ & $64.13 \pm 10.41$ & -0.770 & 0.451 \\
Body mass index $\left(\mathrm{kg} / \mathrm{m}^{2}\right)$ & $23.02 \pm 5.46$ & $24.97 \pm 5.91$ & -0.776 & 0.454 \\
Skeletal muscle mass & $24.66 \pm 5.89$ & $22.27 \pm 5.75$ & 0.917 & 0.371 \\
Body fat mass & $15.20 \pm 8.30$ & $23.03 \pm 9.23$ & -10.993 & 0.062 \\
Strength & $19.64 \pm 9.37$ & $16.63 \pm 8.59$ & 0.749 & 0.464 \\
Flexibility & $-1.98 \pm 13.17$ & $6.88 \pm 8.14$ & -10.800 & 0.087 \\
Sit-up & $16.70 \pm 11.59$ & $12.30 \pm 10.44$ & 0.892 & 0.384 \\
Step-test & $100.8 \pm 9.42$ & $116.70 \pm 10.00$ & -10.540 & 0.140 \\
Self-efficacy & $27.81 \pm 3.39$ & $28.17 \pm 4.29$ & -0.206 & 0.839 \\
\hline
\end{tabular}

Values are presented as mean \pm standard deviation. EG, exercise group; $C G$, control group. 
proved by the Institutional Review Board at Seoul National University for the use of human participants in research (approval number: 1312/001-008). A consent form was distributed and read to the participants outlining the purpose, benefits, and potential risks of participation. Detailed information about the participants is provided in Table 1 of the results section.

\section{Research procedure}

The present study was conducted to investigate the effects of exercise training on community dwelling adults with ID, in cooperation with an Intellectual Disability Welfare Center in South Korea. In consideration of the exercise program schedule, the exercise and CGs were recruited separately. After completing recruitment, pretests of self-efficacy and physical fitness of all participants were conducted before starting the intervention. Baseline data were analyzed with an independent $t$-test for independent samples to determine if an initial difference existed between the groups. After confirming homogeneity between the two groups, the exercise group participated in an exercise program using band exercises and rhythmic activities performed in 90-min sessions, twice weekly for 12 weeks. The CG did not participate in the exercise program. Posttests were conducted after completing the program. All data were treated as quantitative data and analyzed by IBM SPSS ver. 18.0 (IBM Co., Armonk, NY, USA). Two-way analysis of variance with repeated measure was used to analyze the treatment effects. The level of significance was set at $P<0.05$.

\section{Intervention}

The exercise program developed for the current study was designed upon reviewing current literature and then consulted with two experts in adapted physical activity field to meet the abilities and needs of the participants. The exercise program consisted of two parts: aerobic exercise and strength training. The programs were conducted twice a week for 12 weeks, and were $90 \mathrm{~min}$ in duration. Each session consisted of a warm-up, a group-based exercise activity, and a cool-down period. The subjects performed 10 min of stretching for the warm-up and cool down portion of the exercise program to improve flexibility and prevent injuries during the main group-based exercise session. The aerobic exercise consisted of a rhythmic activity with background music. The background music selected ranged from children's songs to Korean pop in consideration of the participants' preferences and the exercise tempo. The intensity of the aerobic exercise program was set by the Borg Rating of Perceived Exertion Scale: RPE 9-11 for weeks 1-4, RPE 11-13 for weeks 4-8, and RPE 12-14 for weeks
Table 2. Exercise program

\begin{tabular}{ll}
\hline Program & \multicolumn{1}{c}{ Contents of exercise } \\
\hline Warm up & \\
Type & Gymnastics \\
Time & 5 min \\
Aerobic exercise & \\
Intensity & RPE 9-14 \\
Type & Rhythm exercise \\
Time & 30 min \\
Rest & 10 min \\
Band exercise & \\
Intensity & $10-15$ RM \\
Type & Crunch, reverse fly, biceps curl, lateral raise, chest press, \\
& squat \\
Time & 30 min \\
Cool down & \\
Type & Gymnastics \\
Time & 5 min \\
\hline
\end{tabular}

RPE, rating of perceived exertion; RM, repetition maximum.

8-12. The resistance exercises were performed using the TheraBand, in the following order: crunches, reverse fly, bicep curl, lateral raise, chest press, and squat. The intensity of the exercises gradually increased from mild to moderate. Detailed information about the exercise program is outlined in Table 2.

\section{Measurements}

\section{Health related physical-fitness}

Cardio pulmonary endurance was measured by the 3-min step test. Each participant was asked to step-up (right foot, then left foot) and then down (right foot, then left foot) from a platform that was raised $30 \mathrm{~cm}$ off the ground. The subjects continued the stepping motion at a quick but comfortable pace for $3 \mathrm{~min}$. After 3 min, the subjects were asked to sit down, and their pulse was counted via the radial artery for $1 \mathrm{~min}$. The count was recorded as their recovery heart rate.

Body composition was measured by Bio-electric Impedance Analysis (BIA) equipment (Inbody 370, Inbody, Cerritos, CA, USA). Participants were asked to stand on the metal foot pads of the machine, then the bioelectrical impedance measurement of body composition was performed through a small alternative current. This is a widely used method to assess body composition. The margin of error derived from BIA is about $3 \%$ to $4 \%$ compared to the skinfold caliper (Kim and Park, 2002).

Muscle endurance was assessed by a sit up test, in which the subjects lay on their backs with knees flexed at a right angle and hands placed on the back of the neck. The subjects continually sat 
up to touch their knees with their elbows for $30 \mathrm{sec}$.

Muscle strength was determined using a digital squeeze dynamometer (T.K.K 5401, Takei, Niigata, Japan). The measurement was carried out twice for each hand, and the highest figure was recorded.

Flexibility of the lower back and hamstrings was measured using the sit-and-reach test. The research participants were asked to sit on the floor with their legs stretched straight ahead and to place the soles of their feet against the sit-and-reach box. The participants were asked to correct their posture, extend their arms, and place their hands on the top of the box, where a measuring tape was placed. Next, the participants were instructed to slide their hands forward slowly on the measuring tape as far as possible. This procedure was repeated twice, and the best results recorded in centimeters were used.

\section{Self-efficacy}

Self-efficacy was assessed using the modified version of physical self-efficacy scale developed by Ryckman et al. (1982). This scale was comprised of two subscales, which represent the subdomains of perceived physical competence and physical self-expression confidence. The Cronbach alpha of the original scale was 0.80 (perceived physical competence 0.85 , physical self-expression confidence 0.69), while the modified scale obtained the Cronbach alphas of 0.79 and 0.68 for perceived physical competence and physical self-expression, respectively. The scale consisted of 14 items. Each response was scored from zero to three, where zero score is not confident and three score is very confident. Higher scores indicated greater self-efficacy. Considering that the study participants were adults with ID, the assessment of self-efficacy was conducted through utilization of an interview, in which the primary researcher read and explained the questions to each participant and recorded their responses when the interviewer was confident that the participants fully understood what was being asked.

\section{PAlevel}

In this study, Accelerometers (GT3X+, ActiGraph, Pensacola, FL, USA) were used to measure the change of PA level, average kcals per day, average moderate to vigorous physical activity (MVPA) per day, daily average of sedentary hours, and average steps, after partaking in the exercise program. The accelerometer is an appropriate measurement tool for people with ID since there is no recall demand. Other previous studies have effectively applied motion sensors for participants with ID (Stanish et al., 2006). Temple and Walkley (2003) demonstrated the validity of energy expenditure calculated via accelerometer reported PA by conducting research on people with mild to moderate ID. The level of PA was measured for 1 week before and after the exercise program. All data were analyzed by the previously reported standard of Dixon-Ibarra et al. (2013), in which an accelerometer was used to assess the level of PA for older adults with ID.

\section{RESULTS}

This study was conducted to investigate the effects of an exer-

Table 3. The change of health related physical fitness

\begin{tabular}{|c|c|c|c|c|c|c|}
\hline \multirow{2}{*}{ Variable } & \multicolumn{2}{|c|}{$E G(n=10)$} & \multicolumn{2}{|c|}{$C G(n=10)$} & \multicolumn{2}{|c|}{ Time $\times$ group } \\
\hline & Pre & Post & Pre & Post & $F$ & $P$-value \\
\hline \multicolumn{7}{|l|}{ Sex } \\
\hline Men & \multicolumn{2}{|c|}{7} & \multicolumn{2}{|c|}{3} & & \\
\hline Women & \multicolumn{2}{|c|}{3} & \multicolumn{2}{|c|}{7} & & \\
\hline Weight (kg) & $59.79 \pm 14.46$ & $59.56 \pm 13.83$ & $64.13 \pm 10.41$ & $63.38 \pm 11.03$ & 0.546 & 0.470 \\
\hline Body mass index $\left(\mathrm{kg} / \mathrm{m}^{2}\right)$ & $23.02 \pm 5.46$ & $23.08 \pm 5.30$ & $24.97 \pm 5.91$ & $24.72 \pm 6.15$ & 1.054 & 0.318 \\
\hline Skeletal muscle mass & $24.66 \pm 5.89$ & $24.96 \pm 6.00$ & $22.27 \pm 5.75$ & $21.79 \pm 5.15$ & 3.052 & 0.078 \\
\hline Body fat mass & $15.20 \pm 8.30$ & $14.37 \pm 7.83$ & $23.03 \pm 9.23$ & $23.07 \pm 10.45$ & 0.625 & 0.440 \\
\hline Strength & $19.64 \pm 9.37$ & $20.13 \pm 6.82$ & $16.63 \pm 8.59$ & $16.32 \pm 9.05$ & 0.248 & 0.625 \\
\hline Flexibility & $-1.98 \pm 13.17$ & $1.63 \pm 11.83$ & $6.88 \pm 8.14$ & $8.08 \pm 5.36$ & 0.827 & 0.357 \\
\hline Sit-up & $16.70 \pm 11.59$ & $20.40 \pm 11.39$ & $12.30 \pm 10.44$ & $12.50 \pm 8.48$ & 5.692 & $0.028^{*}$ \\
\hline Step-test & $100.8 \pm 9.42$ & $116.70 \pm 10.00$ & $111.2 \pm 19.07$ & $120.10 \pm 18.34$ & 2.124 & 0.162 \\
\hline
\end{tabular}

Values are presented as mean \pm standard deviation.

EG, exercise group; $C G$, control group.

${ }^{*} P<0.05$. 
cise program on the self-efficacy and health-related physical fitness of adults with ID. The results were as follows. Two participants in the exercise group stopped attending the class due to personal reasons, and one member of the CG was unavailable for posttesting. These participants were therefore dropped from the analysis. Additionally, two participants in the experimental group (EG) and four in the CG were excluded from the final analysis for failing to meet wear time validity to measure PA level. As a result, the data from eight participants in the EG and six in the CG were included in the final analysis for PA level.

\section{Homogeneity of the participant characteristics}

The characteristics of the participants related to the variables in this study are presented in Table 1 . There were no statistically significant differences in testing the homogeneity between the EG and the CG. Thus, the researchers concluded that initial differences did not exist in the data.

\section{Changes of health-related physical fitness}

Table 3 shows the changes of health-related physical fitness. Af- ter the 12-week exercise program, significant differences were found between the groups for muscle endurance $(P<0.05)$. Though, it was not statistically significant, the EG also displayed an increase in skeletal muscle following the exercise program, while the CG displayed a decrease.

\section{Change of self-efficacy}

The changes in self-efficacy are shown in Table 4. After the 12week exercise program, significant differences were found between the groups in self-efficacy. Specifically, a significant difference $(P<0.05)$ was observed in the subscale of perceived physical ability, but not in physical self-presentation confidence. Thus, this result indicated that the total self-efficacy of the participants was attributed to the perception of improved physical function by participation in the exercise program.

\section{Change of PA level}

Table 5 shows the variables related with PA level (average kcals per day, MVPA per day, daily average of sedentary hours, average steps), which significantly improved in the EG compared to the CG.

Table 4. The change of self-efficacy

\begin{tabular}{|c|c|c|c|c|c|c|}
\hline \multirow{2}{*}{ Self-efficacy } & \multicolumn{2}{|c|}{$E G(n=10)$} & \multicolumn{2}{|c|}{$C G(n=10)$} & \multicolumn{2}{|c|}{ Time $\times$ group } \\
\hline & Pre & Post & Pre & Post & $F$ & $P$-value \\
\hline \multicolumn{7}{|l|}{ Sex } \\
\hline Men & \multicolumn{2}{|c|}{7} & \multicolumn{2}{|c|}{3} & & \\
\hline Women & \multicolumn{2}{|c|}{3} & \multicolumn{2}{|c|}{7} & & \\
\hline Perceived physical ability & $27.81 \pm 3.39$ & $32.10 \pm 4.01$ & $28.17 \pm 4.29$ & $28.65 \pm 2.97$ & 6.467 & $0.020^{*}$ \\
\hline Physical self-presentation confidence & $11.26 \pm 2.61$ & $12.91 \pm 2.37$ & $12.44 \pm 2.49$ & $13.85 \pm 2.53$ & 0.037 & 0.850 \\
\hline Total self-efficacy & $27.81 \pm 3.39$ & $32.10 \pm 4.01$ & $28.17 \pm 4.29$ & $28.65 \pm 2.97$ & 6.326 & $0.022^{*}$ \\
\hline
\end{tabular}

Values are presented as mean \pm standard deviation.

EG, exercise group; CG, control group.

${ }^{*} P<0.05$.

Table 5. The change of physical activity level

\begin{tabular}{|c|c|c|c|c|c|c|}
\hline \multirow{2}{*}{ Variable } & \multicolumn{2}{|c|}{$\mathrm{EG}(\mathrm{n}=8)$} & \multicolumn{2}{|c|}{$\mathrm{CG}(\mathrm{n}=6)$} & \multicolumn{2}{|c|}{ Timex group } \\
\hline & Pre & Post & Pre & Post & $F$ & $P$-value \\
\hline \multicolumn{7}{|l|}{ Sex } \\
\hline Men & \multicolumn{2}{|c|}{5} & \multicolumn{2}{|c|}{1} & & \\
\hline Women & \multicolumn{2}{|c|}{3} & \multicolumn{2}{|c|}{5} & & \\
\hline Average kcals per day (kcal) & $78.37 \pm 37.18$ & $105.77 \pm 53.42$ & $67.86 \pm 31.53$ & $62.25 \pm 20.70$ & 4.905 & $0.047^{*}$ \\
\hline Average MVPA per day (min) & $43.29 \pm 21.59$ & $55.11 \pm 26.52$ & $29.83 \pm 14.13$ & $21.51 \pm 12.79$ & 8.078 & $0.015^{*}$ \\
\hline Daily average of sedentary hour (hr) & $14.77 \pm 1.62$ & $13.50 \pm 1.91$ & $13.66 \pm 2.32$ & $14.45 \pm 2.91$ & 7.763 & $0.016^{*}$ \\
\hline Average steps (count) & $6.67 \pm 2.09$ & $8.27 \pm 2.01$ & $5.30 \pm 1.26$ & $4.61 \pm 2.47$ & 5.617 & $0.035^{*}$ \\
\hline
\end{tabular}

Values are presented as mean \pm standard deviation.

EG, exercise group; CG, control group; MVPA, moderate to vigorous physical activity. ${ }^{*} P<0.05$. 


\section{DISCUSSION}

The purpose of this study was to examine the effects of an exercise intervention on the level of PA, self-efficacy and health-related physical fitness of adults with ID. There are limited studies in the literature confirming changes in the PA levels and self-efficacy in adults with ID following an exercise intervention based on SCT.

Examination of the physical fitness showed an increase in the skeletal muscle mass in the exercise group but a decrease in the CG, though these trends were not statistically significant. However, muscle endurance was significantly improved following the exercise program. Conversely, no significant changes in body composition, strength, flexibility, or cardiovascular endurance were observed after the 12-week exercise intervention.

The findings of this research regarding health-related physical fitness are partly congruent with the study of Calders et al. (2011), where a 20-week exercise program led to significant improvement in muscle endurance, strength and cardiovascular endurance of adults with ID. Similar findings were also reported in Wu et al. (2010) who conducted a 6-month exercise intervention with adults with ID and reported decreases in the participants' weight, BMI scores and categories and positive improvements in the sit and reach test and 30- and 60-sec sit-ups tests. The results of Wu et al. (2010) differ from the observations of the present study. A plausible explanation for this is that the period and intensity of the exercise intervention carried out in this study were insufficient to observe intended changes in the measures, which may also be attributed to differences in the measurement tools used in previous studies.

One of the major findings of the current study is that a significant improvement in self-efficacy was observed following the exercise program in adults with ID. This result was obtained through measurement of the subscale of perceived physical competence, rather than physical self-expression. This revealed that the exercises had an influence not only on physical areas, but also with psychological factors (Biddle and Mutrie, 2007). Self-efficacy was positively changed by recognition of improvement in physical function and mastery experience during the intervention. The results of this study are congruent with those of previous studies. Song and Jeong (2011) reported significant improvement in the self-efficacy and self-esteem of adolescents with ID after 24 weeks of aerobic and strength exercise programs.

Self-efficacy is directly associated with PA behavior and a key factor of SCT (Allen, 2004; Bandura, 1997). In general, people who have higher self-efficacy tend to hold more positive attitude toward the effects of exercise, perceive fewer barriers, engage in more self-regulatory behavior and report more PA than people who have lower self-efficacy (Ayotte et al., 2010). Self-efficacy has been consistently shown to be a predictor of the adoption and maintenance of PA behaviour in healthy adults (Kaewthummanukul and Brown, 2006; Rovniak et al., 2002; Sharma et al.,2005). Empirical evidence has further shown self-efficacy to be a mediator of the effects of interventions on objectively evaluated PA behavior (Burke et al.,2008; Darker et al., 2010; Dutton et al., 2009).

Another major result of this research is improved PA level after participation in the exercise program. This result is congruent with previous studies. The study by Kwon et al. (2009), which examined the effects of a music accompanied jump rope intervention program on the PA level of obese children with ID, indicated a 2-fold increase in the PA level of the exercise group compared to the CG. Based on these results, they argued that activating motivation for PA by including musical aspects in the exercise program is very important for individuals with ID. Ulrich et al. (2011) found that months after to learning ride a bicycle, children with Down syndrome demonstrated a decrease in sedentary behavior, an increase in levels of moderate to vigorous activity, and greater ease performing independently in sport contexts.

Based on these previous studies, the improved PA level observed herein can be explained for two reasons. First, the exercise protocol used in this study was designed to be easy to apply in their real environment. Thus, it is possible that the participants of the exercise group may have participated in PA independently after the experiment finished. Another assumption is supported by the study of Peterson et al. (2008) who reported a significant correlation between self-efficacy and PA of adults with ID $(n=152)$. McAuley (1992), examining the role played by physiological, behavioral, and self-efficacy variables in predicting exercise behavior following 5-month exercise program for middle-aged adults, also reported that self-efficacy perceptions appeared to be significant predictors of PA behavior frequency and intensity during the 3 months of the exercise program is that increased self-efficacy of the participants may affect improvement of their PA levels during the one week of daily life after program finished.

The current study has some limitations, such as the small number of adults with ID, the fact that it was not randomly controlled, and the short follow-up duration (1 week). Therefore, future studies of randomized controlled experimental design and long-term follow-up duration (over 1 month) are needed to clearly 
examine the effects of a similar intervention.

\section{CONFLICT OF INTEREST}

No potential conflict of interest relevant to this article was reported.

\section{REFERENCES}

Allen NA. Social cognitive theory in diabetes exercise research: an integrative literature review. Diabetes Educ 2004;30:805-819.

Anderson-Bill ES, Winett RA, Wojcik JR. Social cognitive determinants of nutrition and physical activity among web-health users enrolling in an online intervention: the influence of social support, self-efficacy, outcome expectations, and self-regulation. J Med Internet Res 2011; 13:e28.

Ayotte BJ, Margrett JA, Hicks-Patrick J. Physical activity in middle-aged and young-old adults: the roles of self-efficacy, barriers, outcome expectancies, self-regulatory behaviors and social support. J Health Psychol 2010;15:173-185.

Bandura A. Health promotion by social cognitive means. Health Educ Behav 2004;31:143-164.

Bandura A. Health promotion from the perspective of social cognitive theory. Psychol Health 1998;13:623-649.

Bandura A. Self-efficacy: the exercise of control. New York: WH Freemand \& Co; 1997.

Bandura A. Social foundations of thought and action: a social cognitive theory. Prentice-Hall series in social learning theory. Englewood Cliffs (NJ): Prentice-Hall, Inc; 1986.

Biddle SJ, Mutrie N. Psychology of physical activity: determinants, wellbeing, and interventions. London: Routledge; 2007.

Burke V, Beilin LJ, Cutt HE, Mansour J, Mori TA. Moderators and mediators of behaviour change in a lifestyle program for treated hypertensives: a randomized controlled trial (ADAPT). Health Educ Res 2008; 23:583-591.

Calders P, Elmahgoub S, Roman de Mettelinge T, Vandenbroeck C, Dewandele I, Rombaut L, Vandevelde A, Cambier D. Effect of combined exercise training on physical and metabolic fitness in adults with intellectual disability: a controlled trial. Clin Rehabil 2011;25:1097-1108.

Darker CD, French DP, Eves FF, Sniehotta FF. An intervention to promote walking amongst the general population based on an 'extended' theory of planned behaviour: a waiting list randomised controlled trial. Psychol Health 2010;25:71-88.

Dixon-Ibarra A, Lee M, Dugala A. Physical activity and sedentary behavior in older adults with intellectual disabilities: a comparative study.
Adapt Phys Activ Q 2013;30:1-19.

Dutton GR, Tan F, Provost BC, Sorenson JL, Allen B, Smith D. Relationship between self-efficacy and physical activity among patients with type 2 diabetes. J Behav Med 2009;32:270-277.

Fernhall B, Tymeson GT. Validation of cardiovascular fitness field tests for adults with mental retardation. Adapt Phys Act Q 1988;5:49-59.

Franklin BA, Whaley MH, Howley ET. ACSM's guidelines for exercise testing and prescription. Philadelphia (PA): Lippincott Williams \& Wilkins; 2000.

Glanz K, Rimer BK, Viswanath K, Orleans CT. Health behavior and health education: theory, research, and practice. San Francisco (CA): John Wiley \& Sons; 2008.

Kaewthummanukul T, Brown KC. Determinants of employee participation in physical activity: critical review of the literature. AAOHN J 2006;54:249-261.

Kang YS, Kim DH. A life history study for understanding on the life of individuals with intellectual disability and physical activity. J Adapt Phys Act Exerc 2010;18:41-62.

Kim HS, Park HS. Reliability and validity of bioimpedance body composition analyzer. Korean J Obes 2002;11:389-397.

King D, Mace FC. Acquisition and maintenance of exercise skills under normalized conditions by adults with moderate and severe mental retardation. Ment Retard 1990;28:311-317.

Kwon HJ, Chung JW, Yang HN, Kim YS. The effects of music jump roping on physical activity and physical fitness of obese children with mental retardation. Korean J Physic Educ 2009;48:529-538.

Lotan M, Isakov E, Kessel S, Merrick J. Physical fitness and functional ability of children with intellectual disability: effects of a short-term daily treadmill intervention. ScientificWorldJournal 2004;4:449-457.

McAuley E. The role of efficacy cognitions in the prediction of exercise behavior in middle-aged adults. J Behav Med 1992;15:65-88.

Peterson JJ, Lowe JB, Peterson NA, Nothwehr FK, Janz KF, Lobas JG. Paths to leisure physical activity among adults with intellectual disabilities: self-efficacy and social support. Am J Health Promot 2008;23: 35-42.

Phillips SM, McAuley E. Social cognitive influences on physical activity participation in long-term breast cancer survivors. Psychooncology 2013;22:783-791

Rovniak LS, Anderson ES, Winett RA, Stephens RS. Social cognitive determinants of physical activity in young adults: a prospective structural equation analysis. Ann Behav Med 2002;24:149-156.

Ryckman RM, Robbins MA, Thornton B, Cantrell P. Development and validation of a physical self-efficacy scale. J Pers Soc Psychol 1982;42: 891-900.

Sharma M, Sargent L, Stacy R. Predictors of leisure-time physical activity 
among African American women. Am J Health Behav 2005;29:352359 .

Song KY, Jeong YT. Effects of combined exercise on self-esteem and physical self-efficacy in the intellectual disability with Down syndrome. Spec Educ Res 2011;10:177-195.

Stacey FG, James EL, Chapman K, Courneya KS, Lubans DR. A systematic review and meta-analysis of social cognitive theory-based physical activity and/or nutrition behavior change interventions for cancer survivors. J Cancer Surviv 2015;9:305-338.

Stanish HI, Temple VA, Frey GC. Health-promoting physical activity of adults with mental retardation. Ment Retard Dev Disabil Res Rev 2006;12:13-21.
Temple VA, Walkley JW. Physical activity of adults with intellectual disability. Am J Intellect Dev Disabil 2003;28:342-353.

Ulrich DA, Burghardt AR, Lloyd M, Tiernan C, Hornyak JE. Physical activity benefits of learning to ride a two-wheel bicycle for children with Down syndrome: a randomized trial. Phys Ther 2011;91:1463-1477.

White SM, Wójcicki TR, McAuley E. Social cognitive influences on physical activity behavior in middle-aged and older adults. J Gerontol B Psychol Sci Soc Sci 2012;67:18-26.

Wu CL, Lin JD, Hu J, Yen CF, Yen CT, Chou YL, Wu PH. The effectiveness of healthy physical fitness programs on people with intellectual disabilities living in a disability institution: six-month short-term effect. Res Dev Disabil 2010;31:713-717. 